Research Article

\title{
Simulation Analysis of Void Defect Detection in Sandwich-Structured Immersed Tunnel Using Elastic Wave
}

\author{
Ruiqiang Liu, ${ }^{1,2}$ Songhui Li, ${ }^{1,2}$ Guoxin Zhang $\mathbb{D D}^{1,2}$ Shenyou Song, ${ }^{3}$ and Jianda Xin ${ }^{1,2}$ \\ ${ }^{1}$ State Key Laboratory of Simulation and Regulation of Water Cycle in River Basin, \\ China Institute of Water Resources and Hydropower Research, A-1 Fuxing Road, Beijing 100038, China \\ ${ }^{2}$ Department of Structures and Materials, China Institute of Water Resources and Hydropower Research, A-1 Fuxing Road, \\ Beijing 100038, China \\ ${ }^{3}$ Administration of Shenzhen-Zhongshan Passage, Guangzhou 510600, China
}

Correspondence should be addressed to Guoxin Zhang; zhanggx@iwhr.com

Received 21 April 2021; Accepted 12 August 2021; Published 19 August 2021

Academic Editor: Shuang Li

Copyright (c) 2021 Ruiqiang Liu et al. This is an open access article distributed under the Creative Commons Attribution License, which permits unrestricted use, distribution, and reproduction in any medium, provided the original work is properly cited.

Void defects can be easily generated between a steel plate and concrete joint due to the complicated internal structure of a sandwich-structured immersed tunnel (SSIT), which affect the overall bearing capacity of the main structure of the immersed tube tunnel. A prototype experiment was conducted to study the application of impact imaging method in the nondestructive detection of void defects in SSITs. The detection criterion for the impact imaging method was established based on the features of the waveform data. Nevertheless, the influence of steel plate thickness, material properties, void location, and structure on the detection accuracy of the impact imaging method is unclear. Therefore, numerical simulation was applied to study the influencing factors by establishing a different condition model. Good agreement between the experimental and numerical results was observed for the response waveform collected from the inspection area. Using the calculation model and identified material parameters validated in the active prototype experiment, numerical simulations of several sets, which considered all influencing factors, were performed. The application scope and sensitivity of the impact image method were recommended to reduce misjudgement in practical applications and improve detection accuracy.

\section{Introduction}

Sandwich-structured immersed tunnels are essential components in the field of cross-sea tunnel structural engineering which have a complicated internal structure. The void defects between the contacting surface of the steel and concrete reduce the carrying capacity of the structure. A fullscale model prototype experiment was conducted to study the application of the impact imaging method in the nondestructive detection of void defects in steel shell concrete. This experiment is an evolution of the traditional model test [1], which enables the consideration of the steel shell structure, size, concrete casting, and other factors. According to the actual construction conditions, construction process, and construction methods of a sandwich-structured immersed tunnel (SSIT) [2-4], fullscale test was conducted to study the void defects that may occur during the actual production and construction [5-7]. As a result of the full-scale model experiment, the detection criterion for the impact imaging method was determined through the features of the waveform data collected from compartments of the full-scale model, and blind tests on the other 12 compartments were performed to study the prediction precision of this method.

Nevertheless, in this experiment, the influence of steel plate thickness, material properties, void position, and structure on the detection results of the impact imaging method was unclear, and the propagation process of elastic 
waves is abstract [8-11]. Therefore, a numerical analysis based on prototype experiments is necessary. Finite element numerical simulation models of various defects were established to validate and extend the prototype experiment. This analysis consisted of identification of the influences of the steel plate thickness, void position, void size, and floating layer on the detection results of the impact image method to study the application range and sensitivity of this detection method.

\section{Model: Wave Equation}

For the stress state at a certain point in the elastic body, a small hexahedral differential body should be cut out near that point. The edges of the body are parallel to the $x$-, $y$-, and $z$-axes, respectively. The outer normal vector in the same direction as the $x$-, $y$-, and $z$-axes is defined to be positive. Then, the stress components on the three negatives are $\sigma_{x}$, $\sigma_{x y}$, and $\sigma_{x z}$ on the $x$-axis; $\sigma_{y}, \sigma_{y x}$, and $\sigma_{y z}$ on the $y$-axis; and $\sigma_{z}, \sigma_{z x}$, and $\sigma_{z y}$ on the $z$-axis. The stress components on the three front faces are [12]

$$
\begin{aligned}
& x \text {-axis front: } \sigma_{x}+\partial \sigma_{x} / \partial x \mathrm{~d} x, \tau_{x y}+\partial \tau_{x y} / \partial x \mathrm{~d} x, \tau_{x z}+ \\
& \partial \tau_{x z} / \partial x \mathrm{~d} x \\
& y \text {-axis front: } \sigma_{y}+\partial \sigma_{y} / \partial y \mathrm{~d} y, \tau_{y x}+\partial \tau_{y x} / \partial y \mathrm{~d} y, \tau_{y z}+ \\
& \partial \tau_{y z} / \partial y \mathrm{~d} y \\
& z \text {-axis front: } \sigma_{z}+\partial \sigma_{z} / \partial z \mathrm{~d} z, \tau_{z x}+\partial \tau_{z x} / \partial z \mathrm{~d} z, \tau_{z y}+\partial \tau_{z y} \\
& / \partial z \mathrm{~d} z
\end{aligned}
$$

The balance equation of the differential body is

$$
\sum F_{(X, Y, Z)}=0 .
$$

The balanced differential equation is

$$
\left\{\begin{array}{l}
\frac{\partial \sigma_{x}}{\partial x}+\frac{\partial \sigma_{y x}}{\partial y}+\frac{\partial \sigma_{z x}}{\partial z}+F_{x}=0 \\
\frac{\partial \sigma_{y}}{\partial y}+\frac{\partial \sigma_{x y}}{\partial x}+\frac{\partial \sigma_{z y}}{\partial z}+F_{y}=0 \\
\frac{\partial \sigma_{z}}{\partial z}+\frac{\partial \sigma_{y z}}{\partial y}+\frac{\partial \sigma_{x z}}{\partial x}+F_{z}=0
\end{array}\right.
$$

According to the summing convention expression

$$
\sigma_{i j, j}=\frac{\partial \sigma_{i x}}{\partial x}+\frac{\partial \sigma_{i y}}{\partial y}+\frac{\partial \sigma_{i z}}{\partial z} \quad(i=x, y, z),
$$

the equation can be rewritten as

$$
\sigma_{i j, j}+F_{i}=0 \quad(i=x, y, z) .
$$

The geometric equation of three-dimensional strain in space is

$$
\left\{\begin{array}{l}
\varepsilon_{x}=\frac{\partial u}{\partial x}, \\
\varepsilon_{y}=\frac{\partial v}{\partial y}, \\
\varepsilon_{z}=\frac{\partial w}{\partial z}, \\
\gamma_{x y}=\frac{\partial v}{\partial x}+\frac{\partial u}{\partial y} \\
\gamma_{y z}=\frac{\partial w}{\partial y}+\frac{\partial v}{\partial z} \\
\gamma_{z x}=\frac{\partial u}{\partial z}+\frac{\partial w}{\partial x}
\end{array}\right.
$$

In the isotropic uniform constitutive equation

$$
\left\{\begin{array}{l}
\varepsilon_{x}=\frac{1}{E}\left[\sigma_{x}-\mu\left(\sigma_{y}+\sigma_{z}\right)\right] ; \gamma_{y z}=\frac{\tau_{y z}}{2 G}, \\
\varepsilon_{y}=\frac{1}{E}\left[\sigma_{y}-\mu\left(\sigma_{z}+\sigma_{x}\right)\right] ; \gamma_{z x}=\frac{\tau_{z x}}{2 G}, \\
\varepsilon_{z}=\frac{1}{E}\left[\sigma_{z}-\mu\left(\sigma_{x}+\sigma_{y}\right)\right] ; \gamma_{x y}=\frac{\tau_{x y}}{2 G},
\end{array}\right.
$$

where

$$
G=\frac{E}{2(1+\mu)} .
$$

Here, $E$ is the modulus of elasticity, $\mu$ is Poisson's ratio, and $G$ is the shear modulus.

Equation (6) is the basic equation of elasticity in equilibrium. However, under dynamic conditions, the displacement $U$ is not only a function of the point $(x, y, z)$, but also a function of the time $t$. Therefore, equation (5) is substituted into equation (6) and then into equation (2), thereby obtaining the dynamic displacement equation

$$
\left\{\begin{array}{l}
(\lambda+\mu) \frac{\partial}{\partial x}\left(\frac{\partial u_{x}}{\partial x}+\frac{\partial u_{y}}{\partial y}+\frac{\partial u_{z}}{\partial z}\right)+\mu \nabla^{2} u_{x}=\rho \frac{\partial^{2} u_{x}}{\partial t^{2}} \\
(\lambda+\mu) \frac{\partial}{\partial y}\left(\frac{\partial u_{x}}{\partial x}+\frac{\partial u_{y}}{\partial y}+\frac{\partial u_{z}}{\partial z}\right)+\mu \nabla^{2} u_{y}=\rho \frac{\partial^{2} u_{y}}{\partial t^{2}} \\
(\lambda+\mu) \frac{\partial}{\partial z}\left(\frac{\partial u_{x}}{\partial x}+\frac{\partial u_{y}}{\partial y}+\frac{\partial u_{z}}{\partial z}\right)+\mu \nabla^{2} u_{z}=\rho \frac{\partial^{2} u_{z}}{\partial t^{2}}
\end{array}\right.
$$


and Laplacian

$$
\nabla^{2}=\frac{\partial^{2}}{\partial x^{2}}+\frac{\partial^{2}}{\partial y^{2}}+\frac{\partial^{2}}{\partial z^{2}} .
$$

When $\nabla=\partial / \partial x+\partial / \partial y+\partial / \partial z$, the equation is rewritten as [2]

$$
\begin{aligned}
(\lambda+\mu) \nabla \nabla u+\mu \nabla^{2} u & =\rho \frac{\partial^{2} u}{\partial t^{2}} \\
\text { or }(\lambda+\mu) u_{i j, j}+\mu u_{i j, j} & =\rho u .
\end{aligned}
$$

Here, $u$ indicates displacement, and $\lambda, \mu$, and the Lamé constant can be expressed by the following equation:

$$
\begin{aligned}
& \lambda=\rho\left(V_{L}^{2}-2 V_{S}^{2}\right), \\
& \mu=\rho V_{S}^{2},
\end{aligned}
$$

where $V_{L}$ indicates the longitudinal wave velocity, and $V_{S}$ indicates the shear wave velocity.

\section{Prototype Experiment}

3.1. Equipment of Impact Imaging Method. The testing equipment consisted of an US GEODE high-resolution seismograph, a small steel hammer vibration exciter, a vertical geophone CDJ-Z100 with a working frequency of 100 hertz, a network adapter, a computer, and related connecting cables; see Figure 1 for the details. The shock elastic wave was excited by a human with a hammer, and the excitation frequency was generally tens to thousands of hertz. During data collection, the shock hammer excitation waveform was recorded at the same time to normalise the response waveform data.

3.2. Full-Scale Model Experiment. A full-scale model was used to study the application of the impact imaging method on the nondestructive detection of void defects in a SSIT. The model was $55.4 \mathrm{~m}$ wide, $10.6 \mathrm{~m}$ high, and $9.6 \mathrm{~m}$ long, and it consisted of 105 compartments: 51 floor compartments, 12 wall compartments, and 42 roof compartments. The fullscale model steel shell weighed approximately $740 \mathrm{t}$, and the poured concrete volume was approximately $1400 \mathrm{~m}^{3}$, as shown in Figure 2(a). The experiment adopted the same construction conditions, process, and method in the actual project to simulate the possible void defects in the SSIT pouring process.

The compartments of the full-scale model were poured with self-compacting concrete. After the initial setting period, the compartment was taken as the testing unit to carry out the impact imaging method void defect detection experiment. When data collection was completed, parts of the compartments of the full-scale model were opened. In them, the steel plate was moved to check the filling situation of the concrete. In this study, the typical test data of the prototype experiment and the numerical simulation results were selected for comparative analysis, and a numerical model under various defect conditions was established to analyse

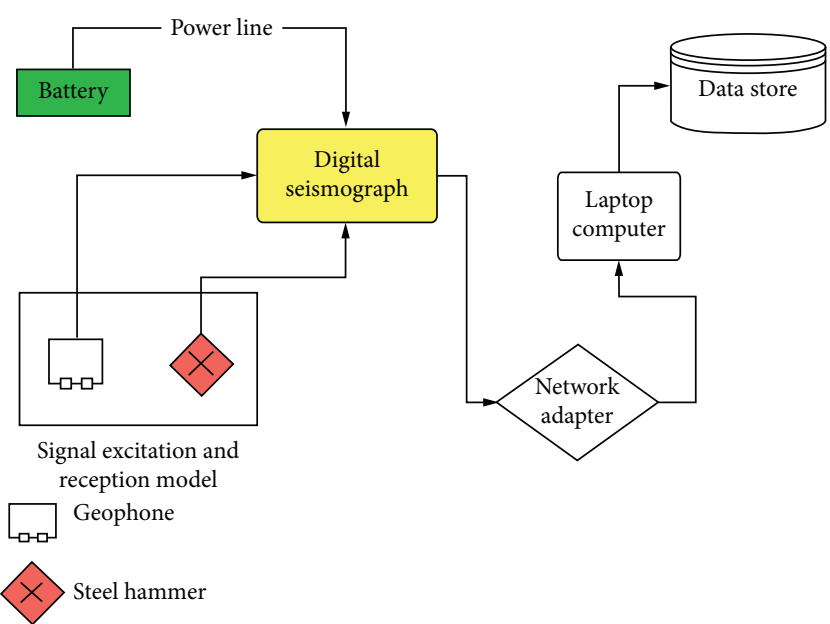

FIgURE 1: Composition of testing equipment.

the factors that affect the detection accuracy of the impact imaging method. The size of the compartment was $2.8 \mathrm{~m} \times 3.0 \mathrm{~m} \times 1.5 \mathrm{~m}$, the middle part of the compartment was arranged with a pouring hole, and 10 exhaust holes were arranged around the compartment, as shown in Figures 2(b) and $2(\mathrm{c})$.

\section{Finite Element Model and Material Parameters}

4.1. Finite Element Model. The object of the numerical analysis model is the compartment of the full-scale model, as shown in Figures 2(b) and 2(c). The numerical models set a rectangular void defect, and the size of the defects is determined by the calculation conditions, such as $200 \mathrm{~mm} \times 20 \mathrm{~mm}$. The model is a two-dimensional finite element model. The mesh used is shown in Figure 3. It consists of a steel plate part $(2.8 \mathrm{~m} \times 0.014 \mathrm{~m})$ and concrete part $(2.8 \mathrm{~m} \times 1.486 \mathrm{~m})$. The impact load is applied from the upper surface of the steel plate, and the dynamic response is analysed. Free boundary conditions are set on the upper surface of the model, and viscoelastic boundary conditions are set on other boundaries [13-15]. Because the stress value generated by the instantaneous impact load in the model is small, it belongs to the problem of small deformation. Therefore, it can be reasonably assumed that the steel and concrete are isotropic linear elastic material, and the Newmark algorithm is selected for calculation $[11,16]$.

4.2. Wave Characteristics and Meshing Size. The setting of the load step is significant to the accuracy of the elastic wave numerical simulation calculation results. A reasonable load step is generally calculated using the following equation [17]:

$$
\Delta t=\frac{1}{20 f_{\max }} .
$$

Here, $f_{\max }$ is the maximum frequency of the impact elastic wave.

In contrast to the simulation, almost all waveforms of elastic waves appear in the experiment. To make the 


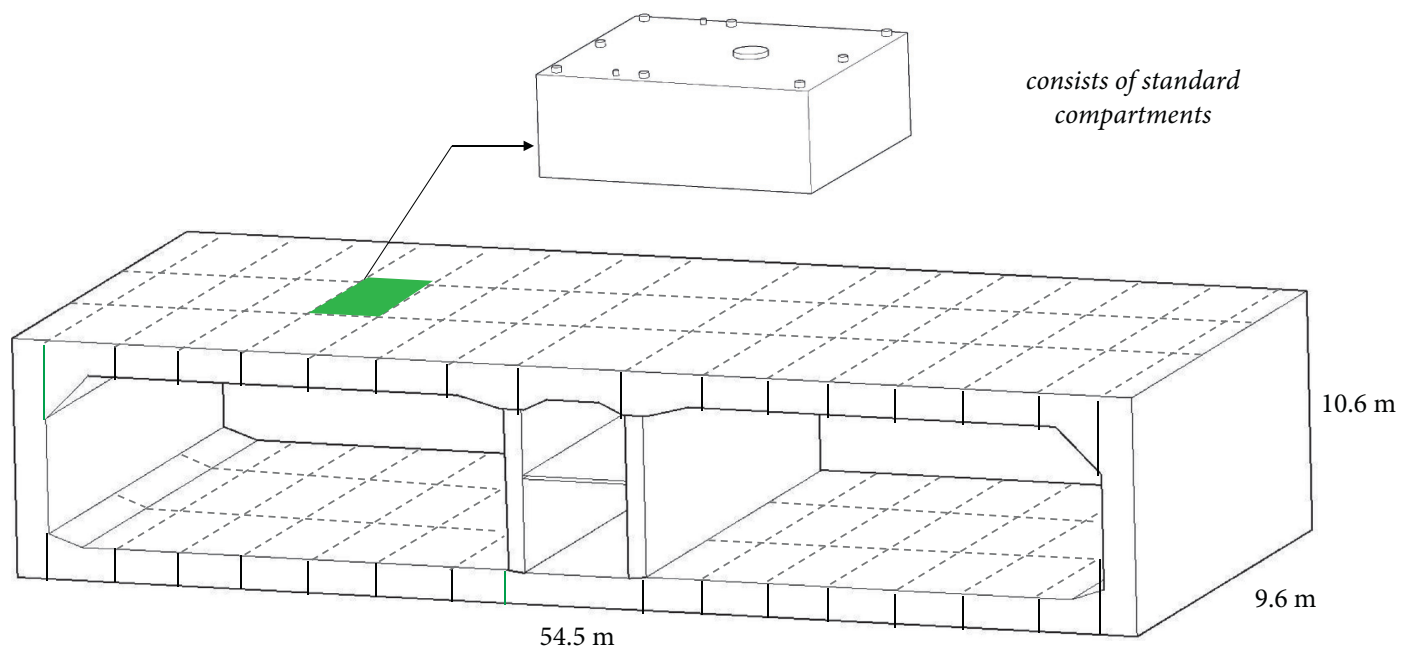

(a)

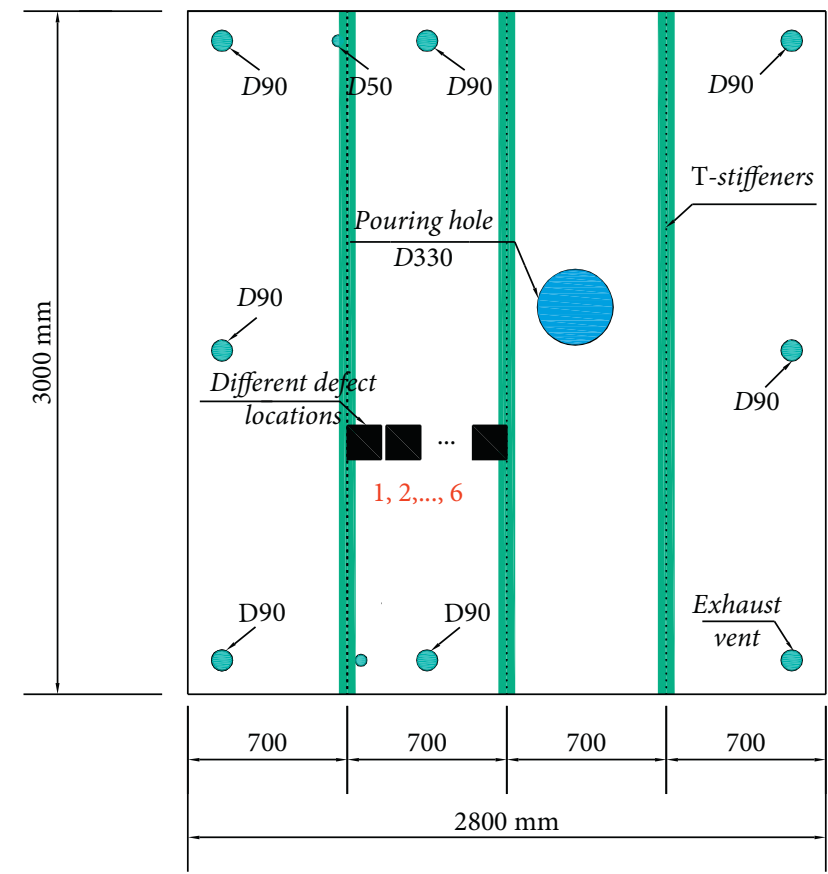

(b)

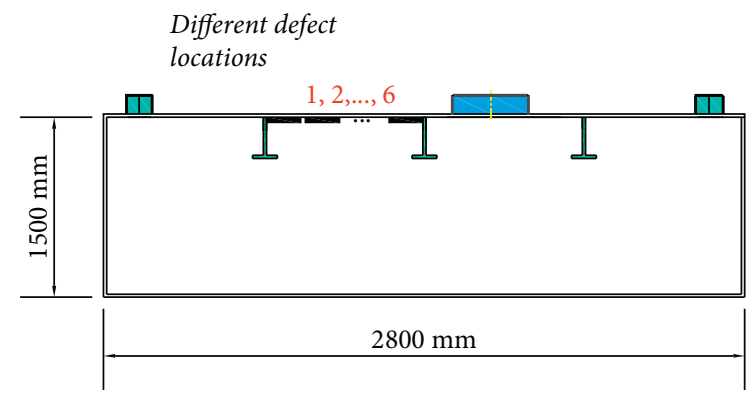

(c)

Figure 2: The structure of experiment model; (a) the full-size SSIT model; (b) the top view of the compartment; (c) the front view of compartment.

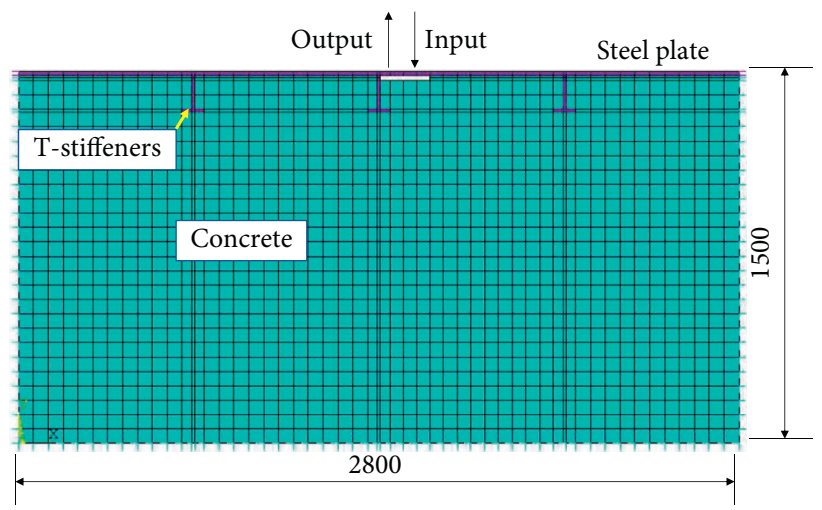

Figure 3: The compartment calculation model of steel shell concrete immersed tube tunnel (mm). 
simulation results closer to the actual situation, each wavelength must contain at least eight nodes [18], and previous research shows that each wavelength containing 10 nodes can meet the simulation precision [19]. Thus, each wavelength contains at least 12 nodes, and the mesh size is calculated by the following equation:

$$
l=\frac{\lambda_{\min }}{12} .
$$

Here, $l$ is the mesh size, and $\lambda_{\min }$ is the minimum wavelength of the impact elastic wave.

According to the experimental waveform data, the frequency range of the elastic wave was $500-2000 \mathrm{~Hz}$. The immersed tube shell was made of Q235 carbon steel; because there is no precise measurement, the propagation speed of its transverse wave was approximately $3200 \mathrm{~m} / \mathrm{s}$, and the longitudinal wave was approximately $5900 \mathrm{~m} / \mathrm{s}$. Therefore, the wavelength of the longitudinal wave was approximately $2.95 \mathrm{~m}$, and the transverse wave was approximately $1.6 \mathrm{~m}$. According to equation (13), the mesh size must be at least $0.13 \mathrm{~m}$.

4.3. Material Properties and Excitation Loads. Owing to the small load and displacement, the material nonlinearity was not considered in this analysis, as shown in Tables 1 and 2. For the weak concrete floating layer (in Section 5.6), the material parameters were first set to the same value as the concrete (see Table 1), and then five calculation conditions were set to reduce the material parameters of the floating layer gradually and study the influence of the weak floating layer on the impact imaging method.

In the experiment, the impact load was excited by the human-operated hammer, which needed to be recorded and normalised. In the simulation analysis, a $0.001 \mathrm{~s}$ half-period triangular load was used to excite the impulse response strength, which was consistent with the impact load carrier shape of the human-operated hammer. The numerical integration took $\Delta t=0.05 \mathrm{~ms}$, and the calculation step length was 1000 steps and was attenuated by the Rayleigh method [20].

\section{Results}

5.1. Wave Characteristics of Void Defect Area. Figure 4 shows the velocity waveform calculated by the finite element numerical simulation, and Figure 5 shows the voltage waveform collected in the experiment. Considering that the thickness of the steel plate was $14 \mathrm{~mm}$ and the width of the void defect was $20 \mathrm{~cm}$ and assuming that the void defect is located near the T-stiffener, the response waveform generally had three peaks corresponding to the test results. Although the ordinate properties of the two waveforms were different, the vibration characteristics (the attenuation tendency of the waveform amplitude and the spectrum characteristics) are basically consistent. Moreover, the results of the spectrum diagram obtained by numerical calculation and the test show that when the elastic wave was excited above the void defect, the peak frequency occurred not only in the low-frequency range of $500-700 \mathrm{~Hz}$, but also in a higher-frequency band, which indicated that the elastic wave encountered the defect in the propagation process and diffraction and reflection occurred, resulting in multiple peaks.

5.2. Wave Characteristics of Dense Area. The results of the waveform (see Figures 6 and 7) show that, in the dense area, the numerical and experimental waveform amplitudes are both small in comparison with the results obtained by the defect area (see Figures 4 and 5). Moreover, the vibration characteristics, such as the attenuation tendency of the waveform amplitude, are extremely consistent. The results of the response waveform spectrogram show that the dense area only has a peak in the range of 500-800 Hz. Compared with the void defect area (see Section 5.1), the main frequency of the dense area is single, showing different vibration characteristics of the two conditions. The comparative analysis of the calculation results in the finite element model and the experimental results illustrates that the calculated waveforms are in excellent agreement with the experimental waveforms. Therefore, the simulation analysis can be used to simulate the propagation process of elastic waves of steel shell concrete.

5.3. Influence of Steel Plate Thickness. In the full-scale model test, only a $14 / 26 \mathrm{~mm}$ steel plate thickness is considered in the fabrication of the steel shell, and the influence of different steel plate thicknesses on the detection results of the impact imaging method is still unclear. Assuming that the void defect is located near the T-stiffener and the defect width is $20 \mathrm{~cm}$ and considering that the impact load is applied to the centre of the defect, in this section, the characteristics of response waveforms under different steel plate thicknesses are compared. In this study, seven models were prepared with the same calculation conditions, except for different steel plate thicknesses. Figure 8 shows that there are two peaks (one at $500-700 \mathrm{~Hz}$ and the other at $1200 \mathrm{~Hz}$ ) in the spectrogram of the void defect, which are quite similar to the results in Section 5.1, although the thicknesses of the steel shell of the models are different.

With the increase of the thickness of steel plate, the vibration characteristics of the defect site change. When the thickness of the steel plate is $8 \mathrm{~mm}$, the frequency peak in the range of $1200 \mathrm{~Hz}$ is quite large compared with other thick steel plate models. As the steel plate thickness increases, the amplitude of the main frequency gradually decreases, and the decrease value is larger in the range of $8-14 \mathrm{~mm}$. As the thickness of steel plate increases, the peak frequency of waveform decreases as low frequency. The analysis results illustrate that the impact imaging method is more sensitive to the void defects under the thin steel plate, and the recognition accuracy is higher. While the thickness of the steel plate increases, the sensitivity to defects decreases, and the recognition accuracy deteriorates. 
TABLE 1: Material parameters.

\begin{tabular}{lccc}
\hline Material type & Elastic modulus $\left(\mathrm{N} / \mathrm{m}^{2}\right)$ & Poisson's ratio & Density $\left(\mathrm{kg} / \mathrm{m}^{3}\right)$ \\
\hline Q235 carbon steel & $2.06 e^{11}$ & 0.28 & 7800 \\
Self-compacting concrete & $3.15 e^{10}$ & 0.24 & 2450 \\
\hline
\end{tabular}

TABle 2: Self-compacting concrete mix proportions.

\begin{tabular}{lcccccccc}
\hline Materials & Cement & Fly ash & Slag powder & $\mathrm{CA}_{1}$ & $\mathrm{CA}_{2}$ & Silica sand & Water & Plasticiser \\
\hline Mix proportions $\left(\mathrm{kg} / \mathrm{m}^{3}\right)$ & 270 & 196 & 84 & 39 & 508 & 782 & 171 & 5.5 \\
\hline
\end{tabular}

FA: fine aggregate, $\mathrm{CA}_{1}$ : coarse aggregate $(5-10 \mathrm{~mm}), \mathrm{CA}_{2}$ : coarse aggregate $(10-20 \mathrm{~mm})$.

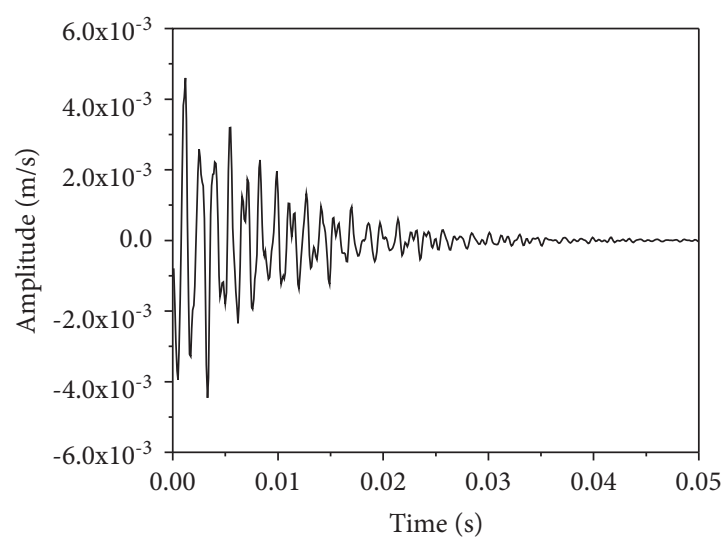

— Fill defect region

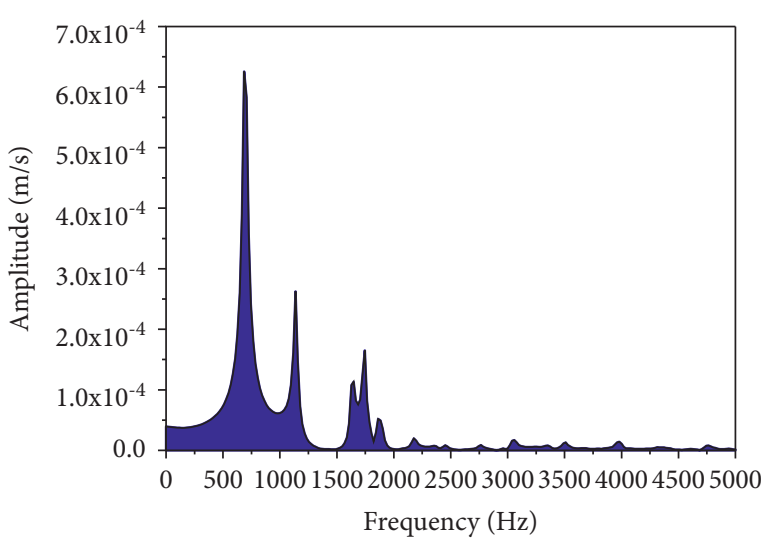

Fill defect region

Figure 4: Time history curve and frequency spectrum of numerical simulation waveform.

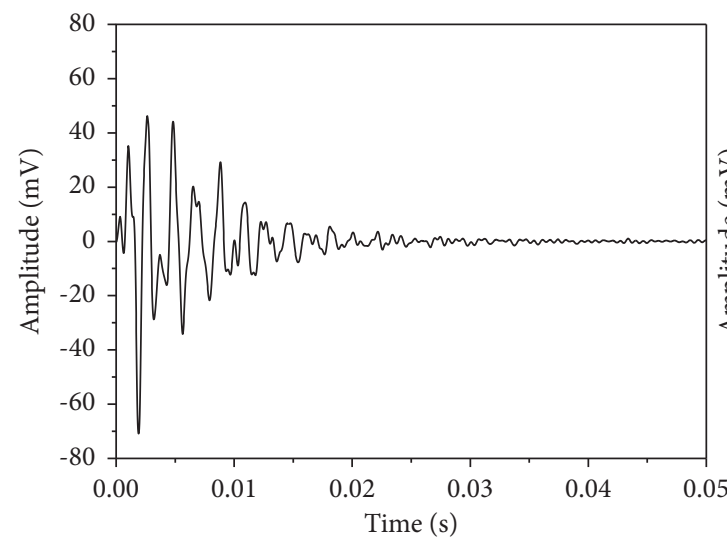

- Fill defect region

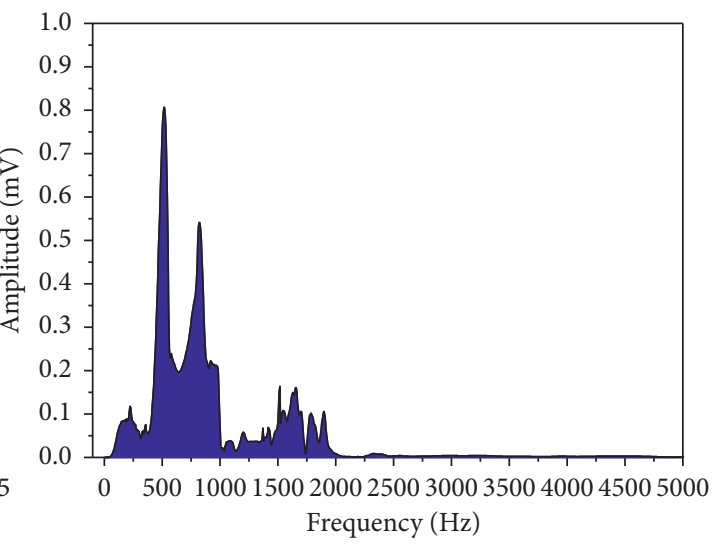

Fill defect region

Figure 5: Time history curve and frequency spectrum of experimental waveform.

5.4. Influence of Void Defect Size. In this section, the sensitivities of the impact imaging method to voids of different sizes when the steel plate thicknesses are 14, 24, and $36 \mathrm{~mm}$ are compared. Figure 9 shows the calculation results of the defect model of the steel plate thickness of $14 \mathrm{~mm}$ with different void defects near the T-stiffener. When the length of the void defect is $25 \mathrm{~cm}$, there is an extremely obvious peak value of the waveform spectrogram in the range of $1100-1200 \mathrm{~Hz}$, which is different from the feature of the dense region. Therefore, it is easy to distinguish the defect. However, with the decrease in the void size, the frequency peak value in the range of $1100-1200 \mathrm{~Hz}$ decreases significantly. When the defect length is $10 \mathrm{~cm}$, the spectrograms of the defect part and the dense part are basically the same. Therefore, in the $14 \mathrm{~mm}$ steel plate thickness model, it is difficult to identify small void defects with a defect length of less than $10 \mathrm{~cm}$.

Figure 10 shows the sensitivity analysis results of the impact imaging method to the defect size under different steel plate thickness models. It can be seen from the figure that when the defect length is $10 \mathrm{~cm}$, the difference in the 


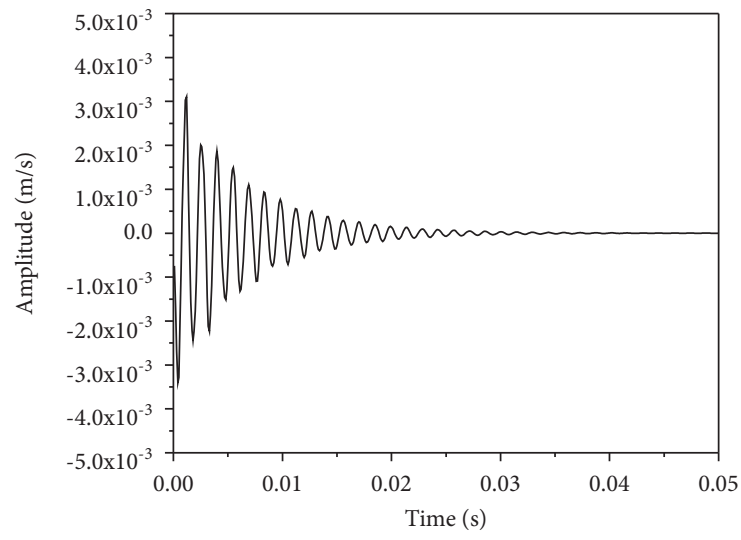

- Fill dense region

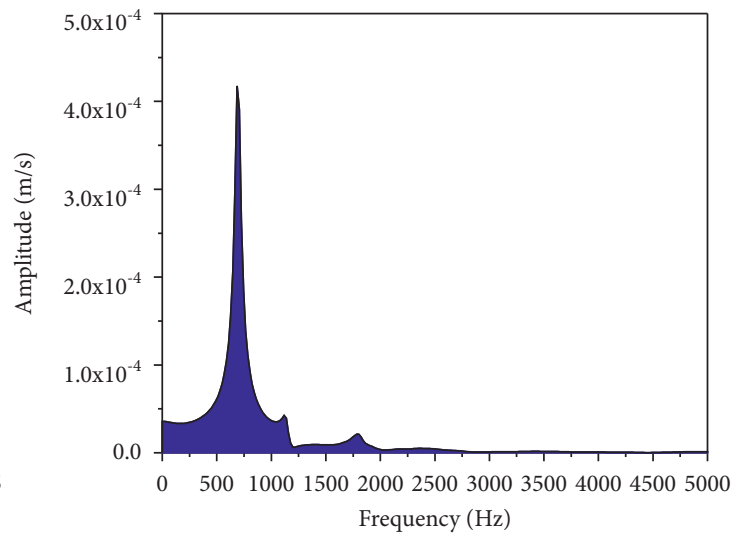

Fill dense region

FIGURE 6: Time history curve and frequency spectrum of numerical simulation waveform.

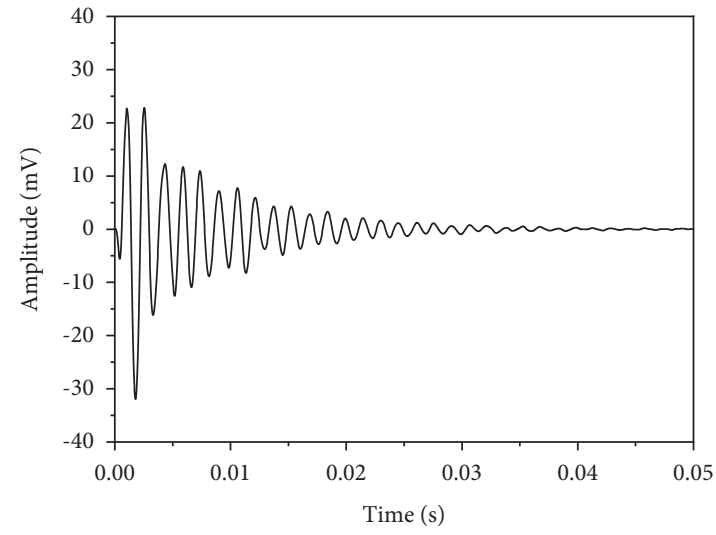

Fill dense region

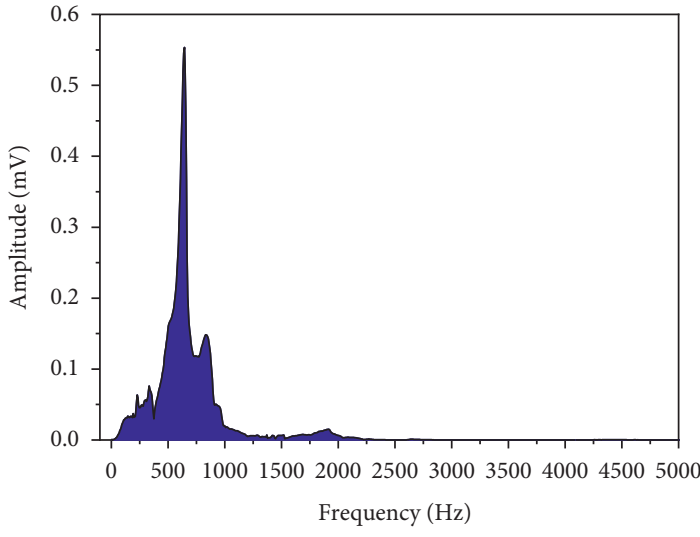

Fill dense region

Figure 7: Time history curve and frequency spectrum of experimental waveform.

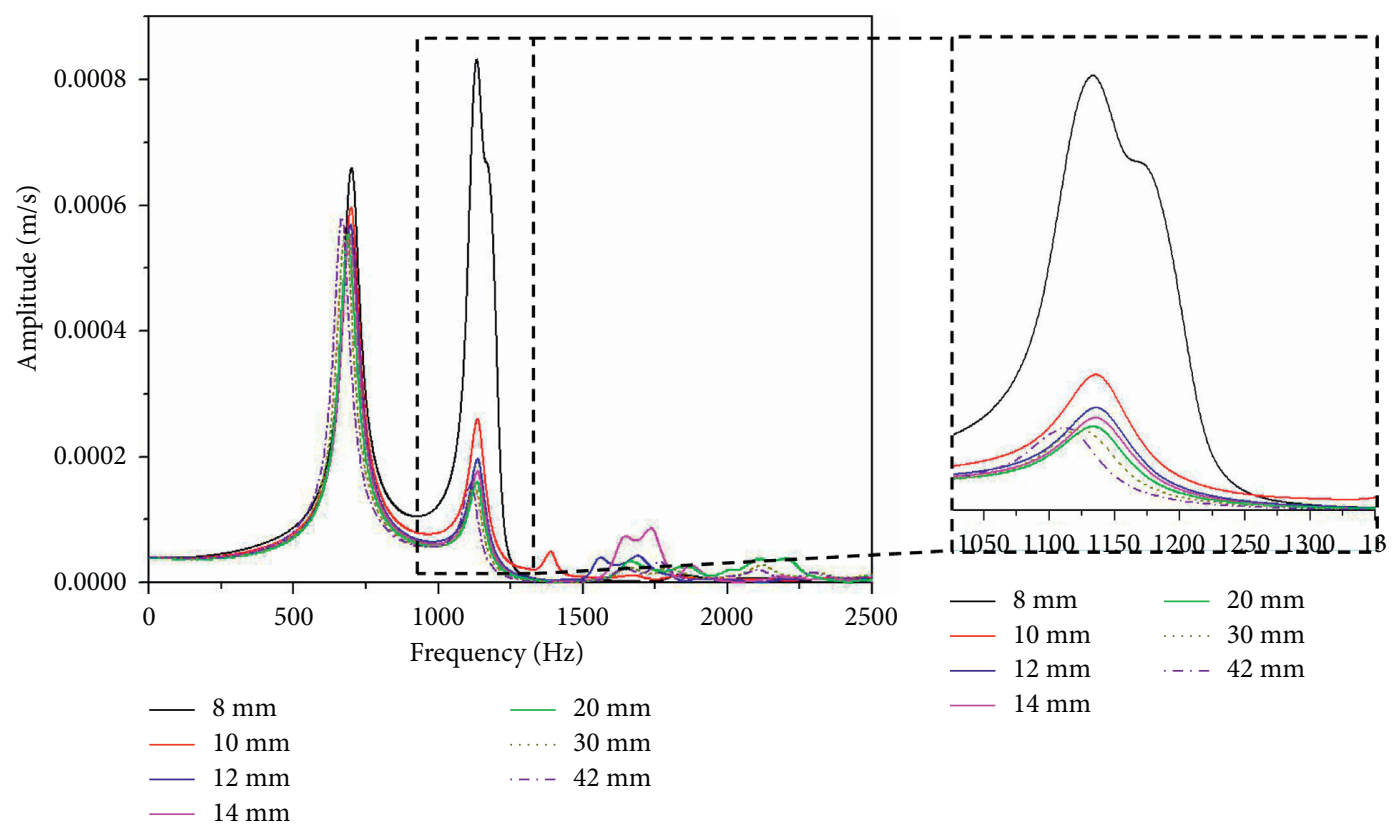

FIGURE 8: Waveform frequency spectrum of void defect area under different steel plate thicknesses. 


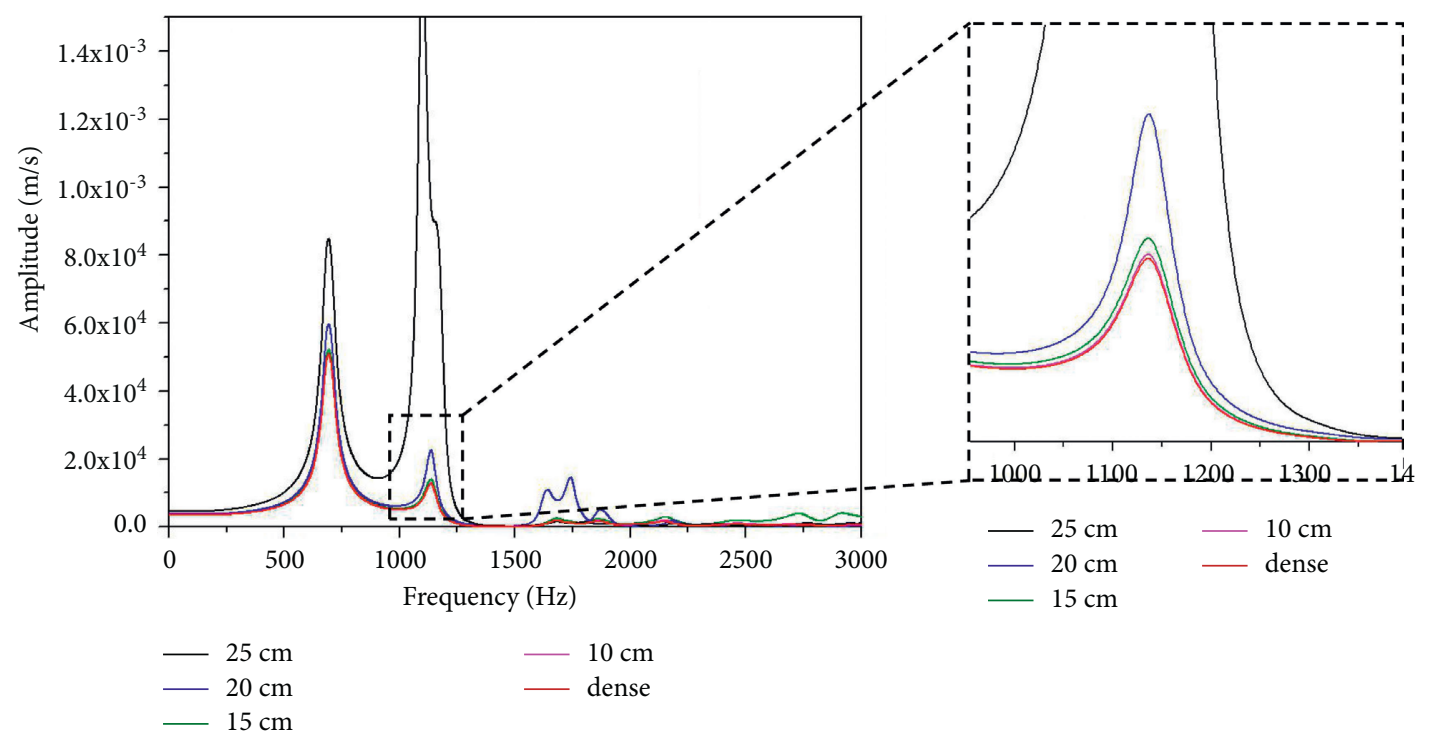

Figure 9: Response waveform frequency spectrum under different defect lengths.

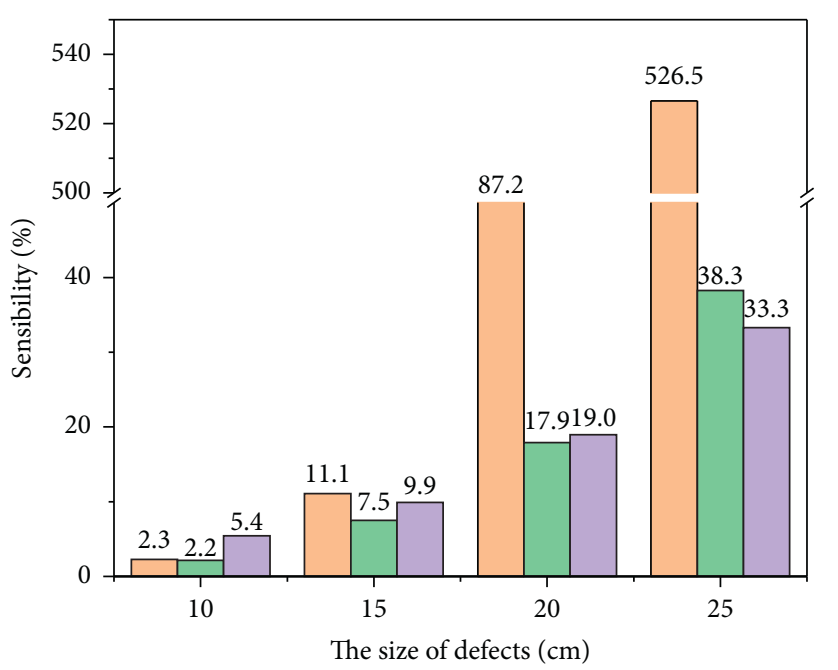

\begin{tabular}{|r|r}
\hline & $14 \mathrm{~mm}$ \\
\hline \hline & $24 \mathrm{~mm}$ \\
& $36 \mathrm{~mm}$
\end{tabular}

FIGURE 10: Response waveform frequency spectrum under different defect lengths.

waveform characteristics between the defect area and the dense area is basically within 5\%, and the detection sensitivity of the three steel plate thickness models is relatively small. When the defect length is $15 \mathrm{~cm}$, the difference is basically close to 10\%; at this time, the void defect can be distinguished. When the defect length is $20 \mathrm{~cm}$, the model of $14 \mathrm{~mm}$ thickness steel plate has a significant increase in difference, reaching $87.2 \%$, and the differences of the $24 \mathrm{~mm}$ and $36 \mathrm{~mm}$ model are basically close to $20 \%$, and the void defect can be easy identified. The results indicate that the impact mapping method is more sensitive to void defects with a defect length greater than $15 \mathrm{~cm}$, while it is less sensitive to void defects below $10 \mathrm{~cm}$, and the recognition accuracy is not high.
5.5. Influence of Excitation-Receiving Location. The theoretical basis of the impact imaging method is elastic wave theory and near-source wave field theory. After the elastic wave field is excited on the surface of the detected object, a detector is placed near the excitation location to receive the wave field information of the detected area. The load excitation position and the location of the geophone are different, resulting in different paths of the elastic wave, which in turn affects all the wavefield information. The arrangement of the geophone and the load excitation position affects the detection accuracy of the impact imaging method, so there are three calculation models-(1) excite and receive above the void defect, (2) excite at the edge of the void defect and receive at the dense position, and (3) excite at the dense area and receive above the void defect (see Table 3 and Figure 11) - to study the ability to identify the void defect by the impact imaging method when the excitation and receiving positions are different.

The results of the different calculation conditions are presented in Figure 12. When stimulating and receiving on the defect region, the received response shows an obvious waveform characteristic of the defect region (the same as in Figure 13). When stimulating the defect area while receiving in the dense area, the characteristics of the received response waveform are the same as the characteristics of the response waveform information in the dense area. When stimulating in the dense area and the detector is placed just above the void defect, the received response waveform shows the waveform characteristics of the defect area. Therefore, the placement of the detector affects the characteristics of the response waveform. When only the detector is placed in the region above the void defect, the received response waveform shows obvious defect response waveform characteristics. However, when the detector is not placed above the defect, although the stimulating position is above the defect, the response waveform characteristics are too similar to the dense area, and it is difficult to detect the void defect. 
TABLE 3: Sensitivity analysis of different excitation-receiving locations.

\begin{tabular}{lcr}
\hline Calculation condition & Excitation-receiving location & Receiving \\
\hline$A$ & Excitation & Defect area \\
$B$ & Defect area & Dense area \\
$C$ & Defect area & Defect area \\
\hline
\end{tabular}

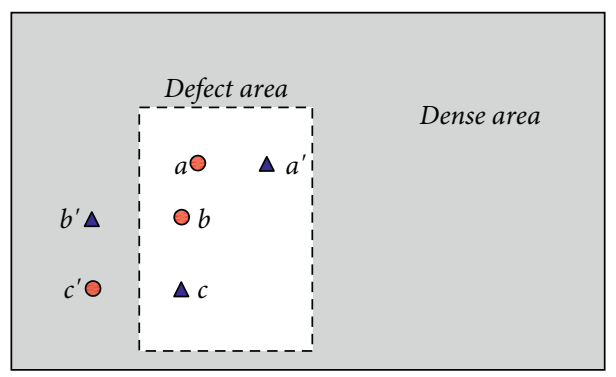

$\Delta$ Receiving location

- Excitation location

FIgURE 11: Excitation and reception positions.

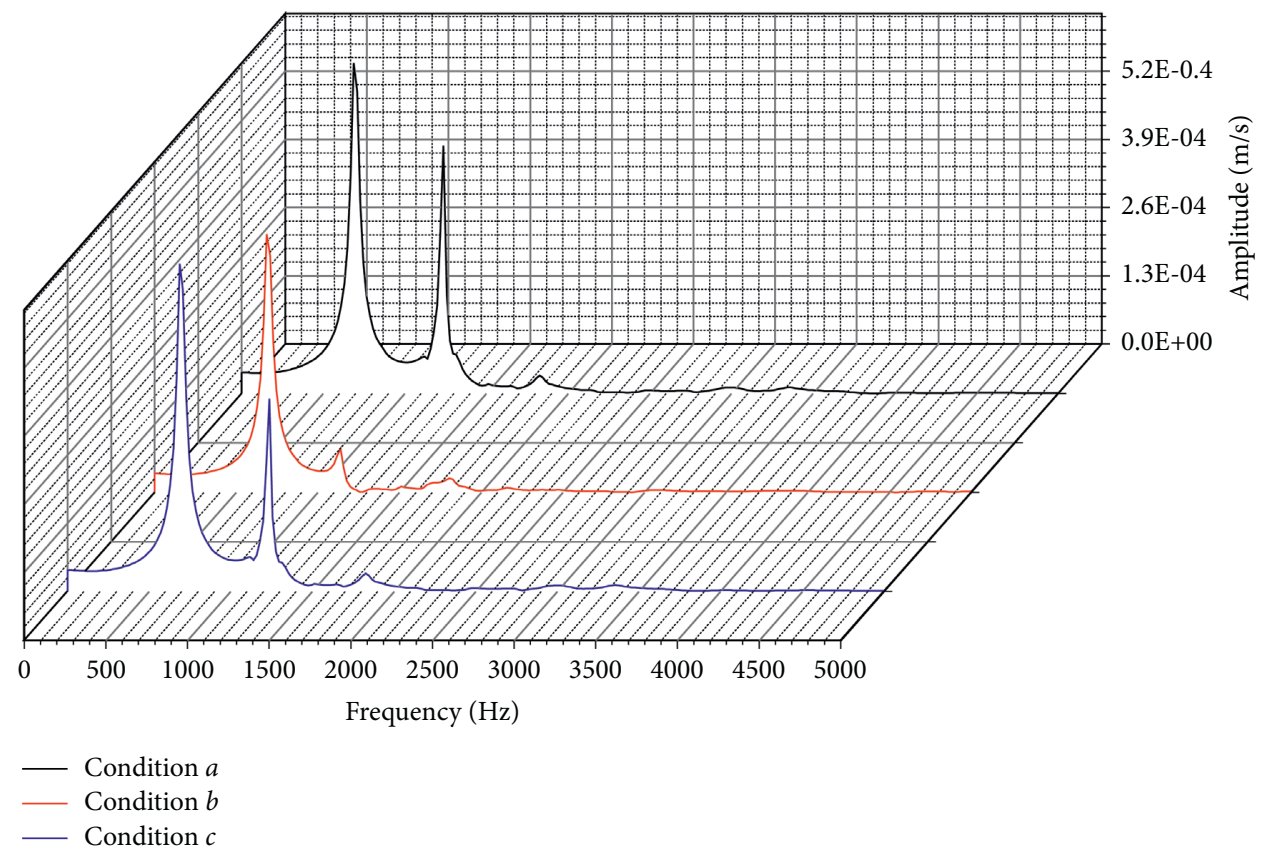

FIgURE 12: Spectrogram of defect area at different tap positions.

This situation should be considered in actual defect void inspection. If the detector is not above the defect, a missed inspection may occur. Therefore, the measurement position should be set reasonably by considering the thickness of the steel plate, the minimum allowable void defect range, and the limitations of the testing equipment.

5.6. Influence of T-Stiffeners Structure. Each compartment of the immersed tube tunnel was poured with self-compacting concrete by reserved pouring holes. Owing to the complicated structure of the interior stiffening T-stiffener and welding nail structure, the void defects located at different positions may have different response waveform characteristics. In this study, 12 models that contain six types of void position between the two T-stiffeners were set up to calculate the waveform characteristics and compare them with the dense situation at the same/corresponding position (see Figures 2(b) and 2(c)).

Although the detected structure positions are different, the waveform characteristics of the dense area at different positions are relatively consistent, and the waveform spectrogram from positions 1-6 has only one peak (see Figure 14). The void defect area's waveform spectrogram from 


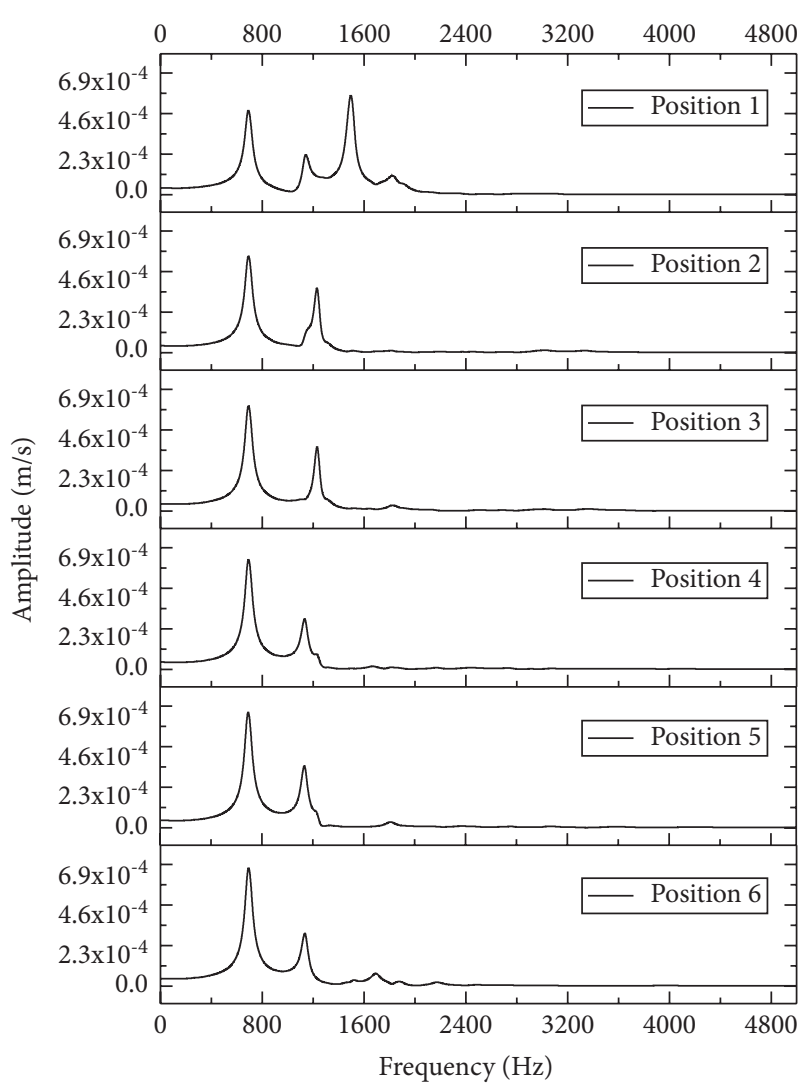

FigURE 13: Waveform spectrum diagram of defect region.

the positions 2-6 has two peaks, and the characteristic waveform is quite consistent (see Figure 13). However, in position 1 which is close to the boundary, three spectral peaks appear in the higher-frequency band (see position 1 in Figure 13), indicating that multiple waves overlap in the void area at this time. Although the size of the void defect is the same, there are certain differences in the vibration characteristics of the different location, but the basic waveform characteristics are the same, which is quite different from the single spectrum characteristics of the dense area. It does not affect the recognition accuracy of the impact mapping method. It can distinguish between the void defect and the filling density at different positions.

5.7. Influence of Concrete Floating Layer. In the blind certification test, it was found that the defect prediction model established by the features of the waveform data collected from the prototype experiments misjudged the dense area to be a void area. However, although the concrete filled under the steel plate was sufficient, the strength of the concrete was significantly lower compared with the design standards, resulting in a weak concrete floating layer. In the casting and hardening process of self-compacting concrete, a $1-2 \mathrm{~cm}$ floating slurry layer may form on the upper layer if the concrete is configured with too high collapse degree or if some water reducing agents are used improperly. The influence of the floating layer on the detection accuracy of the

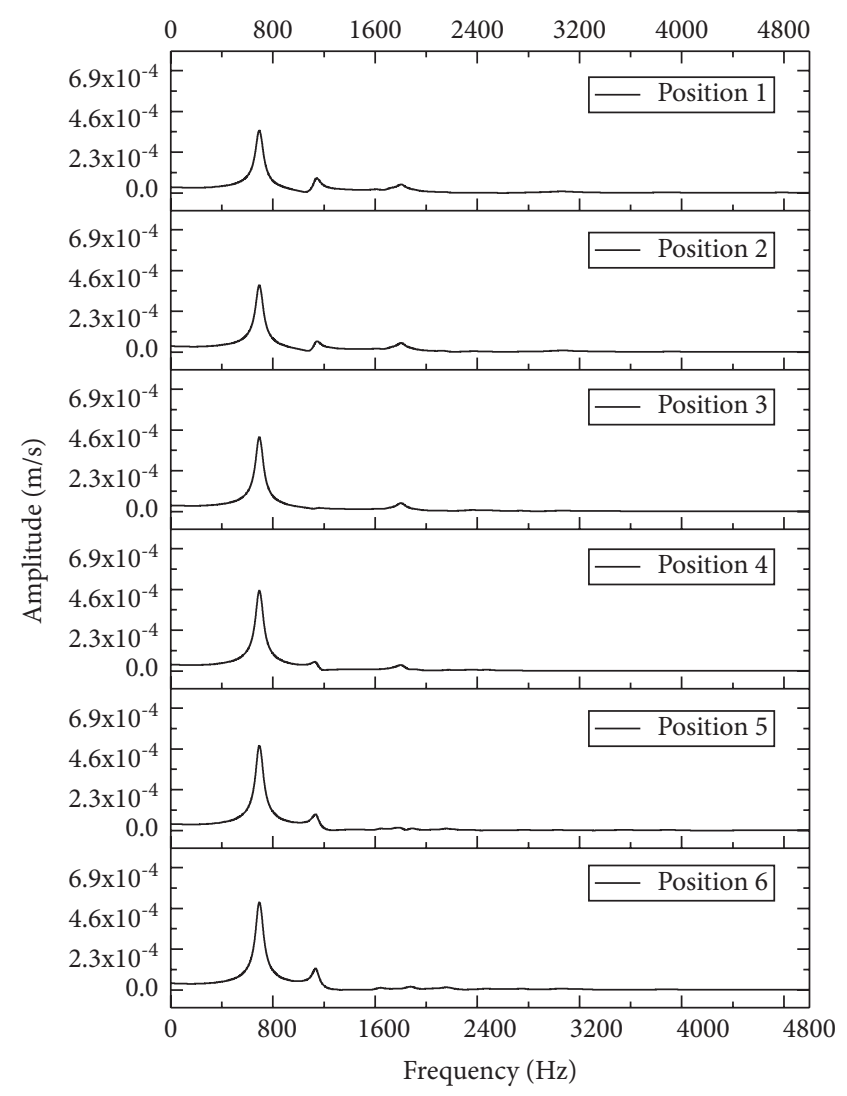

FIgURE 14: Waveform spectrogram of dense region.

impact imaging method was studied by setting the floating slurry layer in the calculation model (see Figure 15). The strength of the floating slurry layer was analysed by gradually decreasing the strength, and six calculation conditions were set, as shown in Table 4.

The calculation results of the gradual decrease of the material parameters under various working conditions reveal that the frequency distribution of the response waveform vibration changes with the change in the material parameters. Considering that the elastic modulus in the material parameters is reduced to $1.00 e^{8} \mathrm{~N} / \mathrm{m}^{2}$ and the density is reduced to $1700 \mathrm{~kg} / \mathrm{m}^{3}$, the characteristics of the response waveform and the frequency spectrum are the same as those collected from the void defect area. As shown in Figure 16, the response waveform has two spectral peaks in the spectrogram-the same features as discussed in Section 5.1 (see Figure 5).

The simulation results show that the existence of the weak interlayer of the concrete floating layer affects the identification results of the impact imaging method. When the strength of the floating layer is low, the impact imaging method can easily identify it as an imperfectly filled void defect area. However, the floating layer generally exists in a large area, so the influence of the floating layer should be considered in the actual void defect detection when a large area of voids exists in the detection result. 


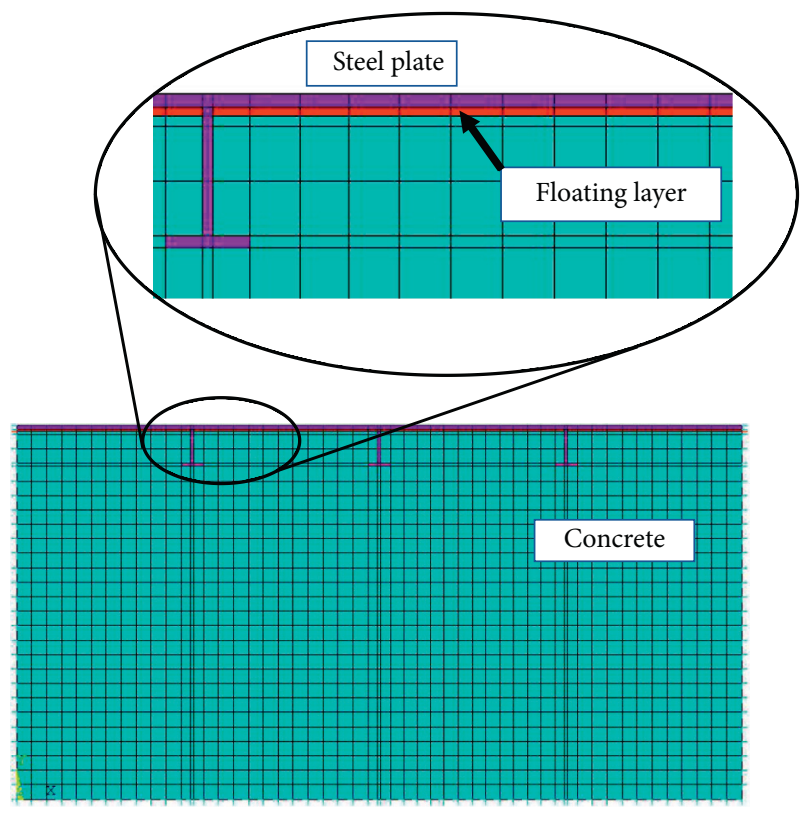

FIGURE 15: Calculation model of concrete floating layer.

TABLE 4: Calculation conditions of material parameters of concrete floating layer.

\begin{tabular}{lccc}
\hline Model & $E\left(\mathrm{~N} / \mathrm{m}^{2}\right)$ & Poisson ratio & Density $\left(\mathrm{kg} / \mathrm{m}^{3}\right)$ \\
\hline 1 & $3.15 e^{10}$ & 0.24 & 2450 \\
2 & $1.00 e^{10}$ & 0.24 & 2200 \\
3 & $3.15 e^{9}$ & 0.24 & 2000 \\
4 & $3.15 e^{8}$ & 0.26 & 1700 \\
5 & $1.00 e^{8}$ & 0.26 & 1700 \\
6 & $3.15 e^{7}$ & 0.26 & 1700 \\
\hline
\end{tabular}

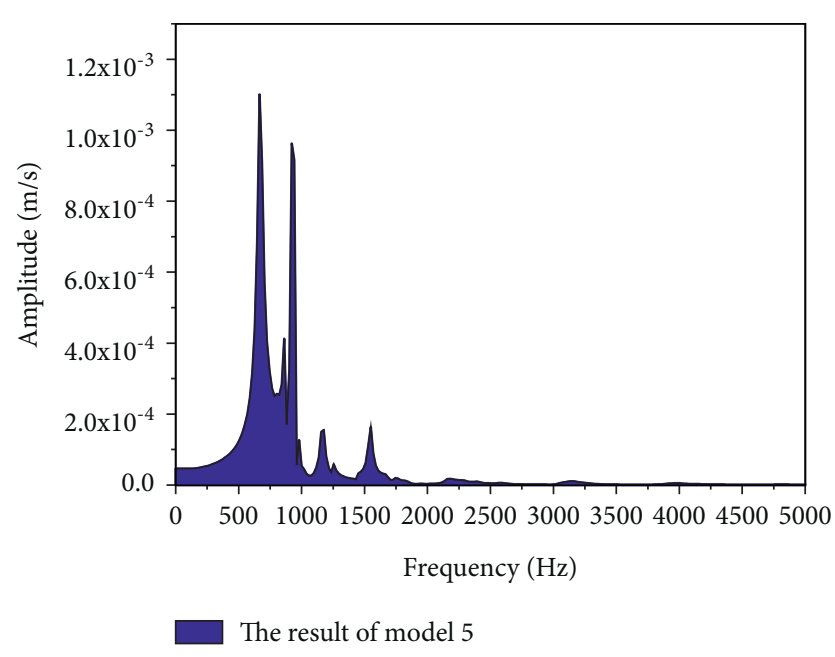

FIgURE 16: Waveform spectrum with floating layer.

\section{Conclusion}

A numerical simulation was performed to verify and extend the prototype experiments. In this paper, the influence of many factors (steel plate thickness, material properties, void location, etc.) on the detection result of the impact imaging method is analysed. The research results are as follows.

(1) The voltage waveform measured in the experiment corresponds well to the surface vibration waveform obtained by the numerical simulation, indicating that the calculation parameters used in the numerical simulation are consistent with the actual situation, and the calculation results are reliable.

(2) As the thickness of the steel plate increases, the peak amplitude of high frequency spectrum of response waveform decreases, indicating that the thinner the thickness of the steel plate (less than $14 \mathrm{~mm}$ ), the better the detection effect and accuracy of the method. The sensitivity analysis results of the defect size show that the impact imaging method is more sensitive to the void defects with a length greater than $15 \mathrm{~cm}$, while it is less sensitive to void defects with a length less than $10 \mathrm{~cm}$.

(3) The numerical results show that when the waveform is excited and received above the void defect, the method can identify the void at different structural positions. However, the placement of the geophone affects the detection effect; when the geophone is not located above the defect, the void defect cannot be identified.

(4) When the floating layer is on the concrete surface in the compartment, the calculated vibration waveform shows obvious waveform characteristics of the void defect, indicating that the existence of the floating layer causes the impact imaging method to misjudge and identify the dense area as a void defect.

Thus, in actual engineering inspection, the measuring lines should be arranged reasonably to ensure that the void defects within the measurable range are not missed. And the floating layer generally appears on a large scale; when a large void area is shown in the detection results, the influence of the floating layer should be considered.

\section{Data Availability}

The data used to support the findings of this study are available from the corresponding author upon request.

\section{Conflicts of Interest}

The authors declare that they have no conflicts of interest.

\section{Acknowledgments}

The authors would like to acknowledge the support provided by the Guangdong Province Key Field R\&D Program Project: key technology of steel Shell concrete immersed tube tunnel construction in complex ocean environment (2019B111105002); National Key R\&D Program (2018YFC0406902); and National Natural Science Foundation of China (52079061). 


\section{References}

[1] H. Rathod and R. Gupta, "Sub-surface simulated damage detection using Non-Destructive Testing Techniques in reinforced-concrete slabs," Construction and Building Materials, vol. 215, no. 10, pp. 754-764, 2019.

[2] W. C. Grantz, "Steel-shell immersed tunnels- Forty years of experience," Tunnelling and Underground Space Technology, vol. 12, no. 1, pp. 23-31, 1997.

[3] A. Glerum, "Developments in immersed tunnelling in Holland," Tunnelling and Underground Space Technology, vol. 10, no. 4, pp. 455-462, 1995.

[4] M. J. Tomlinson, A. Tomlinson, and M. L. Chapman, Immersed Tunnel Techniques, Thomas Telford Publishing, London, UK, 1990.

[5] H. Kimura, H. Moritaka, and I. Kojima, "Development of sandwich-structure submerged tunnel tube production method," Nippon Steel Technical Report, vol. 86, pp. 86-93, 2002.

[6] A. Keiichi, H. Youichi, K. Hitoshi et al., "Immersed tunnels in Japan: recent technological trends," in Proceedings of the 2002 International Symposium on Underwater technology, Tokyo, Japan, April 2002.

[7] S. Song, J. Nie, G. Xu et al., "Development and application of steel-concrete-steel composite structures in immersed tunnels," China Civil Engineering Journal, vol. 52, no. 4, pp. 109-120, 2019.

[8] S. Iyer, S. K. Sinha, B. R. Tittmann, and M. K. Pedrick, "Ultrasonic signal processing methods for detection of defects in concrete pipes," Automation in Construction, vol. 22, pp. 135-148, 2012b.

[9] S. Iyer, S. K. Sinha, M. K. Pedrick, and B. R. Tittmann, "Evaluation of ultrasonic inspection and imaging systems for concrete pipes," Automation in Construction, vol. 22, no. Mar., pp. 149-164, 2012a.

[10] D. Wei, Z. Wu, X. Zhou et al., "Experimental studies on void detection in concrete-filled steel tubes using ultrasound," Construction and Building Materials, vol. 128, no. dec.15, pp. 154-162, 2016.

[11] C. Liu, A.-l. Che, and S.-K. Feng, "Propagation characteristics of elastic wave in layered medium and applications of impact imaging method," Journal of Shanghai Jiaotong University, vol. 18, no. 4, pp. 479-485, 2013.

[12] W. Maocheng, Finite Element method, Tsinghua University Press, Beijing, China, 2003.

[13] A. J. Deeks and M. F. Randolph, "Axisymmetric time-domain transmitting boundaries," Journal of Engineering Mechanics, vol. 120, no. 1, pp. 25-42, 1994.

[14] L. Jingbo, G. Yin, and D. Yixin, "Consistent viscous-spring artificial boundaries and viscous-springboundary elements," Chinese Journal of Geotechnical Engineering, vol. 28, no. 9, pp. 1070-1075, 2006.

[15] J. Lysmer and R. L. Kuhlemeyer, "Finite dynamic model for infinite media," Journal of the Engineering Mechanics Division, vol. 95, no. 4, pp. 859-877, 1969.

[16] Y. Yamashita and O. Kiyomiya, "Finite element analysis for detecting unfilled parts of synthetic members by tapping method," in Proceedings of 58th Annual Scientific Lecture Meeting of the Japan Society of Civil Engineers, Japan, 2003.

[17] ANSYS User's Manual for Revision 5.0, Swanson Analysis Systems, Houston,TX, 1992.

[18] C. Hanxin, S. Kui, K. Chanli et al., "Simulation of Ultrasonic Testing Technique by Finite Element Method," in Proceedings of 2012 Prognostics \& System Health Management Conference, PHM-2012 Beijing, 2012.

[19] D. Alleyne and P. Cawley, "A two-dimensional Fourier transform method for the measurement of propagating multimode signals," Journal of the Acoustical Society of America, vol. 89, no. 3, pp. 1159-1168, 1991.

[20] T. Akiyama, K. Osamu, Y. Yamashita et al., "Analytical consideration of tapping method as a non-destructive inspection method," in Proceedings of Annual Proceedings of Concrete Engineering, p. 26, Salt Lake City, Utah, June 2004. 